\title{
Exemple d'application de la PIV à une étude de génie côtier
}

\author{
Jean-Marc Rousset \\ Research Associate, Fluid Mechanics Group, School of Mech. Eng, The Univ. of \\ Edinburgh, EH9 3JL - Edinburgh (Scotland) \\ courriel:jrousset@eng.ed.ac.uk
}

\section{Résumé}

La vélocimétrie par imagerie de particules (PIV) est une méthode de vélocimétrie basée sur le traitement d'images de particules entraînées par un écoulement. Nous présentons les principes de cette méthode et l'équipement nécessaire à sa mise en œuvre. Plusieurs techniques avancées de traitement des images sont également précisées. Une étude expérimentale des écoulements dans la chambre de dissipation d'un caisson perforé constitue un exemple d'application de la PIV.

\begin{abstract}
Particle Image Velocimetry is a velocimetry technique based on the analysis of fluid particles imaging. Principles of this method are introduce as well as the needed equipment to perform it. Advanced image analysis techniques are also presented. An applied PIV example is supported by an experimental study of perforated caisson dissipation chamber flow pattern.
\end{abstract}

\section{Introduction}

La vélocimétrie par imagerie de particule (ou PIV selon son acronyme anglais) est un procédé de vélocimétrie, relativement récent, basé sur le traitement d'images. Le développement de cette méthode a été soutenu initialement par la recherche en aérodynamique mais la pratique de la PIV s'est néanmoins répandue dans le domaine de l'hydrodynamique et dans le milieu industriel. Technique en plein essor, son perfectionnement et son adaptation à différents problèmes actuels constituent d'ailleurs les objectifs des programmes européens EUROPIV et HYDRIV.

L'usage de la PIV dans le domaine du génie côtier reste cependant inhabituel. Cette technique peut notamment être mise en œuvre au cours d'études de la houle effectuées en laboratoire, en particulier pour déterminer rapidement la cinématique globale des écoulements. Dans les paragraphes qui suivent, nous présentons plus précisément cette technique de vélocimétrie et concluons par un exemple d'application en canal à houle. 


\section{Méthodes optiques de vélocimétrie}

Dans les années 1970, sont apparues plusieurs méthodes de vélocimétrie optiques non-intrusives parmi lesquelles la vélocimétrie Doppler à laser (LDV) et la vélocimétrie par interférométrie de "speckle" (LSV). La LSV, la PIV et la vélocimétrie par suivi de particules (PTV), ces deux dernières méthodes étant apparues dans les années 80, reposent sur un même principe de mesure : la détermination des positions successives de particules, entraînées par un écoulement, à partir de leur enregistrement sur plusieurs images (Adrian, 1991). L'évolution des positions au cours du temps conduit ainsi à reconstituer le champ des vitesses au sein de l'écoulement.

Ces trois méthodes se différencient cependant par la densité de l'ensemencement qu'elles réclament: une très forte concentration pour la LSV, un très faible nombre de particules pour la PTV. La PIV demande une densité moyenne, telle qu'une "identification individuelle des particules" soit possible (Adrian, 1984, Lauterborn, 1984).

\section{Principes de la PIV}

La PIV est une méthode qui peut se décomposer en plusieurs étapes. La première phase correspond à l'illumination des particules de l'écoulement. La deuxième étape est définie par l'enregistrement, sur une ou plusieurs images successives, de la lumière diffusée par ces particules. La troisième et dernière phase est essentiellement une étape de calcul afin de déterminer le déplacement des particules entre chaque image. Cette phase est l'objet de nombreux développements récents car elle constitue en fait le noyau de la PIV.

Une description exhaustive de la PIV dépassant le cadre de cet article, le lecteur intéressé par ces différents aspects pourra se reporter à l'ouvrage de Raffel (Raffel et al., 2001).

\subsection{L'illumination des particules}

Les particules sont illuminées par une nappe lumineuse de faible épaisseur mais de forte intensité. Afin de ne pas perturber l'écoulement, la source de la lumière est généralement placée à l'extérieur de la veine d'essais (avec un canal à houle, par exemple). Dans le cas de moyens de grandes dimensions (bassins de traction, cuve à houle, ...), il est néanmoins possible de générer la nappe lumineuse au sein du fluide en intégrant le dispositif optique dans un bulbe hydrodynamique.

La source lumineuse est habituellement constituée soit d'un laser pulsé Nd:Yag (équipement particulièrement efficace pour la PIV), soit d'un laser à Argon ionisé. Ce dernier type de laser est également employé pour la LDV et donc courant dans les laboratoires. Il génère une lumière de façon continue (avec $\lambda=488$ et $514 \mathrm{~nm}$ ) et il est nécessaire de lui adjoindre un dispositif générant des impulsions, dont un exemple est décrit à la partie 4.1 .

La nappe laser est produite par la diffraction du faisceau laser par une ou plusieurs lentilles cylindriques : un simple barreau cylindrique en verre peut suffire pour un laser Ar-ion mais trois lentilles successives sont nécessaires pour un laser pulsé. 
Une concentration suffisante en particules devant être atteinte, celles-ci sont le plus souvent ajoutées à l'écoulement (par dilution ou injection). Cette concentration est liée aux dimensions de la zone étudiée, à la résolution spatiale du système de mesure et aux vitesses de l'écoulement. La détermination de sa valeur adéquate est donc essentiellement empirique.

L'ensemencement nécessaire à la PIV est différent de celui entre employé pour les simples visualisations. Pour ces dernières, l'objectif est en effet de faire apparaître les structures locales de l'écoulement, quitte à privilégier certaines régions du fluide (ensemencement inhomogène). Au contraire, la PIV requiert un ensemencement homogène (idéalement), les structures de l'écoulement ne devenant visibles qu'avec l'évaluation du champ des vitesses (Westerweel, 1997). Dans l'eau, un ensemencement peu coûteux peut être réalisé avec du pollen de pins ( $\phi$ de 50 à $70 \mu \mathrm{m})$ ou des particules de polyamide $(20-30 \mu \mathrm{m})$. Le plus efficace est cependant celui effectué avec des sphères de verre $(10-20 \mu \mathrm{m})$ qui peuvent être éventuellement recouvertes d'une couche de matériau réfléchissant.

\subsection{L'acquisition des images - PIV ou DPIV ?}

Originellement la PIV a mis en cuvre des méthodes liées à la photographie (appareils, optiques, etc.), le film $35 \mathrm{~mm}$ constituant un support procurant une excellente résolution spatiale à un coût abordable. La détermination du déplacement des particules était alors obtenue, à partir d'une image doublement exposée, soit par digitalisation directe soit par une méthode basée sur les franges de Young (Adrian, 1991). Dans les deux cas, ce traitement demandait la mise en œuvre d'un second laser dit "d'interrogation" (He-Ne) et d'un banc optique adapté. Cette méthode de PIV, éprouvée et d'un coût relativement limité, présente cependant deux inconvénients majeurs : sa durée d'analyse (plusieurs heures pour une seule image), le risque d'une ambiguité sur la direction de la vitesse (problème de l'auto-corrélation).

Les progrès conjoints de l'électronique et de l'informatique ont contribué à l'émergence d'une autre méthode de PIV basée intégralement sur des techniques numériques : la DPIV ou Digital Particle Image Velocimetry (Willert et Gharib, 1991). Cette méthode est maintenant très largement répandue car elle offre la possibilité d'obtenir des résultats très rapidement et parfois de les visualiser au cours de l'essais. Le réglage des expériences est également grandement facilité. Dans la suite, les termes PIV et DPIV seront utilisés indifféremment.

L'emploi d'une caméra numérique simplifie beaucoup le processus d'acquisition et de digitalisation de l'image car celle-ci est directement composée des pixels du capteur CCD. Selon les expériences, la caméra peut être un simple caméscope numérique ou une caméra adaptée à la PIV, cette dernière donnant de meilleurs résultats (de par la qualité du capteur et sa sensibilité par exemple).

\subsection{Principe de détermination du déplacement des particules}

A l'issue de l'étape d'acquisition, nous avons une succession d'images séparées par un intervalle de temps $\Delta t$ et chacune correspondant à une illumination des particules de l'écoulement. 
L'image obtenue est dans un rapport de taille (ou "magnification") $M$ vis à vis de la zone de la nappe laser étudiée. Ce facteur $M$ est déterminé dans les configurations complexes, comme celles rencontrées avec un canal à houle, grâce à une mire graduée placée dans le plan de la nappe. Les effets de perspective et distorsions optiques peuvent généralement être pris en compte par une relation linéaire $(M=x / X=y / Y)$.

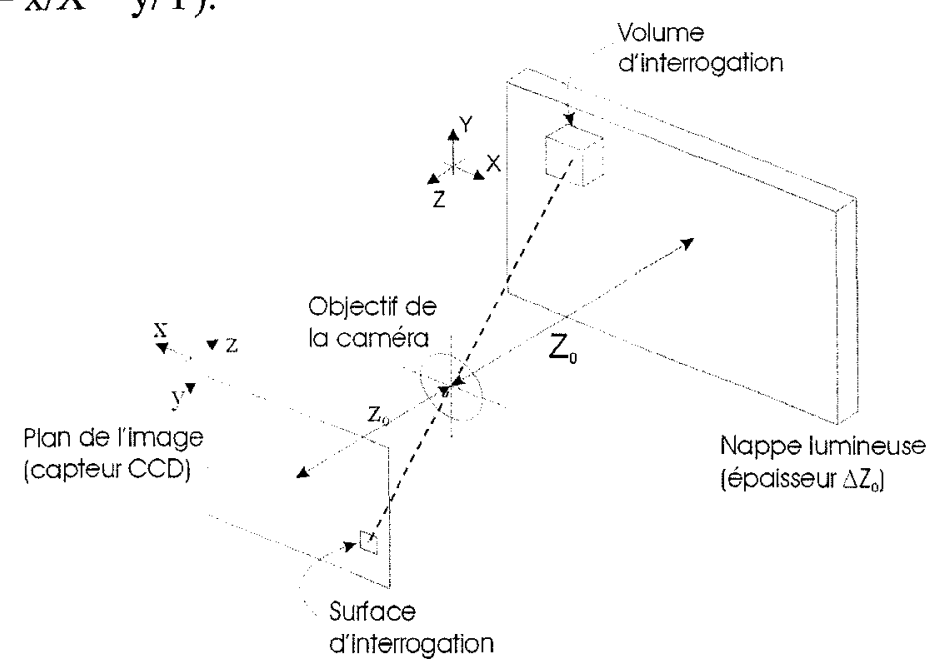

Figure 1. Principe géométrique de la prise de vue du fluide $\left(M=\mathrm{z}_{0} / \mathrm{Z}_{0}\right)$. Geometrical principle of the fluid imaging.

Le déplacement des particules est étudié à l'intérieur d'une portion de l'écoulement illuminé appelé volume d'interrogation, correspondant à une surface d'interrogation dans le plan du capteur de la caméra. Le traitement des images peut donc s'effectuer en découpant l'image en multiples surfaces d'interrogation (fenêtres) et en travaillant uniquement sur des pixels, le retour aux coordonnées réelles $(\mathrm{X}, \mathrm{Y}, \mathrm{Z})$ se faisant à la fin des calculs.

Considérons des particules contenues dans la fenêtre $w_{1}$ de l'image 1 et toujours présentes dans la fenêtre similaire $w_{2}$ de l'image 2 , malgré un déplacement induit par l'écoulement (Figure 2). L'inter-corrélation bidimensionnelle effectuée sur ces deux fenêtres génère une surface présentant un pic. La position de ce pic dans le plan de corrélation peut être comparée à celle que donnerait l'auto-corrélation de $\mathrm{w}_{1}$ : la différence de position (en pixels) des 2 pics correspond au déplacement global des particules reporté dans la fenêtre $w^{\prime}$ du champ estimé des déplacements. En répétant cette opération sur la totalité de l'image en décalant la surface d'interrogation, nous pouvons dresser le champ global déplacement des particules pendant le temps $\Delta t$ puis le champ global des vitesses.

\subsection{Techniques avancées d'évaluation du déplacement des particules}

La méthode précédente est rudimentaire mais elle permet de mettre en évidence les sources d'imprécisions associées à la PIV. Les erreurs potentielles de mesure sont en effet fortement liées au diamètre de la particule, aux différences de contraste des images, aux mouvements du fluide dans le plan de la nappe 
(gradient de vitesse) et traversant ce même plan (vitesse transversale entraînant la disparition de particule dans $\mathrm{w}_{2}$ ).

Plusieurs techniques ont été élaborées afin de prendre en compte les déplacements coplanaires des particules entre $\mathrm{w}_{1}$ et $\mathrm{w}_{2}$ : particules se trouvant hors de $\mathrm{w}_{2}$ ou ayant bougées les une par rapport aux autres.

- Une première méthode consiste déformer la seconde fenêtre d'interrogation. Il est possible de simplement prendre une seconde fenêtre plus grande que la première et d'y chercher les particules (Adrian, 1991). Les méthodes itératives sont plus délicates à mettre en œuvre mais garantissent une plus grande résolution. La première passe est identique à la méthode du 3.3 mais une seconde passe utilise une fenêtre $\mathrm{w}_{2}$ translatée en fonction du déplacement estimé précédemment (Westerweel et al., 1997). La deuxième fenêtre peut également être déformée afin de prendre en compte un gradient de vitesse (Huang et al., 1993).

- Une seconde méthode qui peut être associée avec les précédentes est également itérative. Elle consiste à diminuer la taille de la fenêtre d'interrogation à chaque passe en prenant en compte le déplacement calculé à l'étape précédente. La taille minimale de la fenêtre de la dernière passe (généralement la $3^{\text {ème}}$ ) doit néanmoins contenir au moins 5 paires de particules (5 particules présentes dans $\mathrm{w}_{1}$ et dans $\mathrm{w}_{2}$ ) (Raffel et al, 2001).

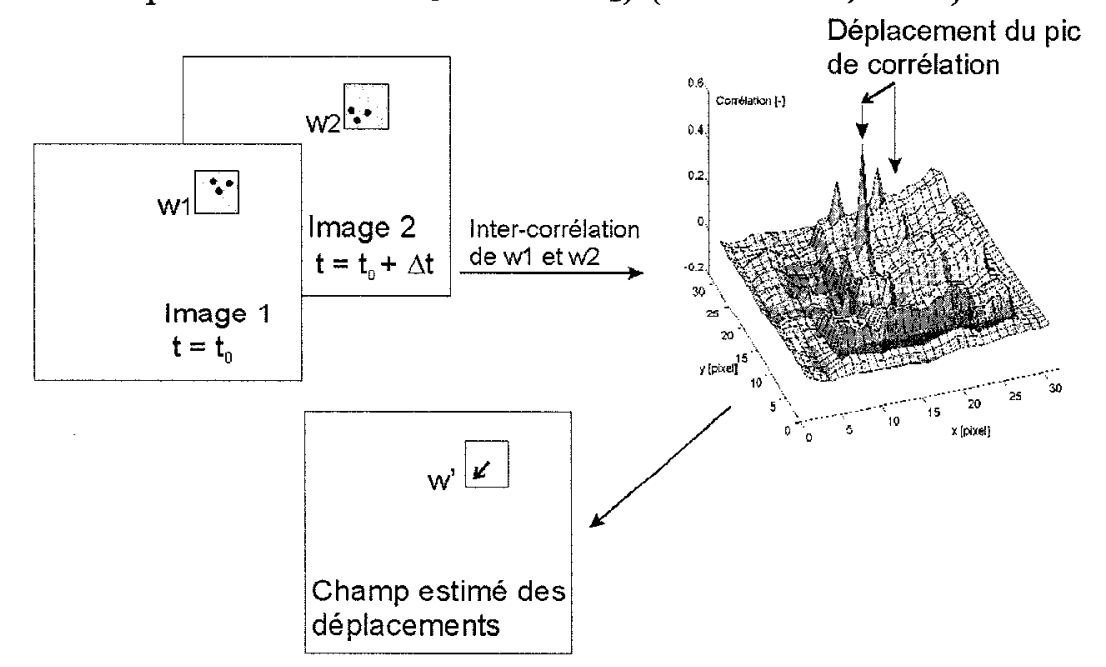

Figure 2. Principe de l'estimation des déplacements des particules par intercorrélation des deux images. Principle of the particles displacement evaluation using image cross-correlation.

Une technique importante et complémentaire des précédentes correspond à l'évaluation du déplacement avec un résolution très inférieure au pixel. Dans la méthode du 3.3, la position du pic de corrélation est déterminée pour une valeur entière de pixels (phénomène de "peak locking") : le déplacement est évalué à $\pm 1 / 2$ px. L'ajustement d'une gaussienne à l'aide de 4 points voisins du pic permet de préciser le déplacement des particules jusqu'au $1 / 20^{\text {ème }}$ de pixel, à la condition que les particules aient un diamètre de 2-3 px (Willert et Gharib, 1991).

A l'issue de ces calculs et de l'obtention du champ des vitesses sur la surface totale de l'image, il reste généralement des vecteurs dont l'orientation ou l'intensité n'ont 
pas de justification physique ("outliers"). Ils peuvent être détectés soit visuellement, soit préférablement par un système de filtres dont les seuils sont déterminés empiriquement. Par exemple, ces valeurs peuvent être basées sur les vitesses maximales ou moyennes présentes dans l'écoulement ou dans le voisinage du point considéré. La valeur erronée est ensuite corrigée par une interpolation des valeurs voisines.

L'étape de validation et de modification des données brutes est très importante et délicate. En effet les calculs postérieurs portant sur l'analyse du champ des vitesses, comme la vorticité, la dissipation d'énergie, etc., sont fortement liés aux méthodes employées lors de cette phase.

\section{Exemple d'application}

Cette partie décrit la mise en œuvre de la PIV dans le cadre d'une étude des écoulements générés par un élément d'une digue à paroi perforée. Nous décrivons les équipements puis la méthode d'analyse.

\subsection{Le dispositif expérimental}

La veine d'essais du canal à houle est entièrement vitrée, large de $0,40 \mathrm{~m}$, longue de $22 \mathrm{~m}$. Le batteur est de type volet oscillant assisté par ordinateur. La maquette schématise un caisson de digue à paroi perforée, constituée d'une paroi frontale portant des fentes horizontales et d'un écran opaque à l'arrière. La largeur de la chambre ainsi constituée est de $0,48 \mathrm{~m}$ (Figure 3 ).

La profondeur d'eau au niveau de la maquette est de $0,30 \mathrm{~m}$, les houles générées sont régulières (périodes $\mathrm{T}=1,3-1,5-1,7-1,9 \mathrm{~s})$ et de même hauteur $(\mathrm{H}=0,05 \mathrm{~m})$. Des sondes limnimétriques ( $\mathrm{S} 1, \mathrm{~S} 2$ et $\mathrm{S} 3$ ) sont placées à $25 \mathrm{~mm}$ des différentes parois de la maquette pour enregistrer les évolutions de la surface libre. La maquette et la plaque horizontale sur laquelle elle est fixée sont en Plexyglass afin d'éviter les zones d'ombre dans la zone illuminée par la nappe laser.

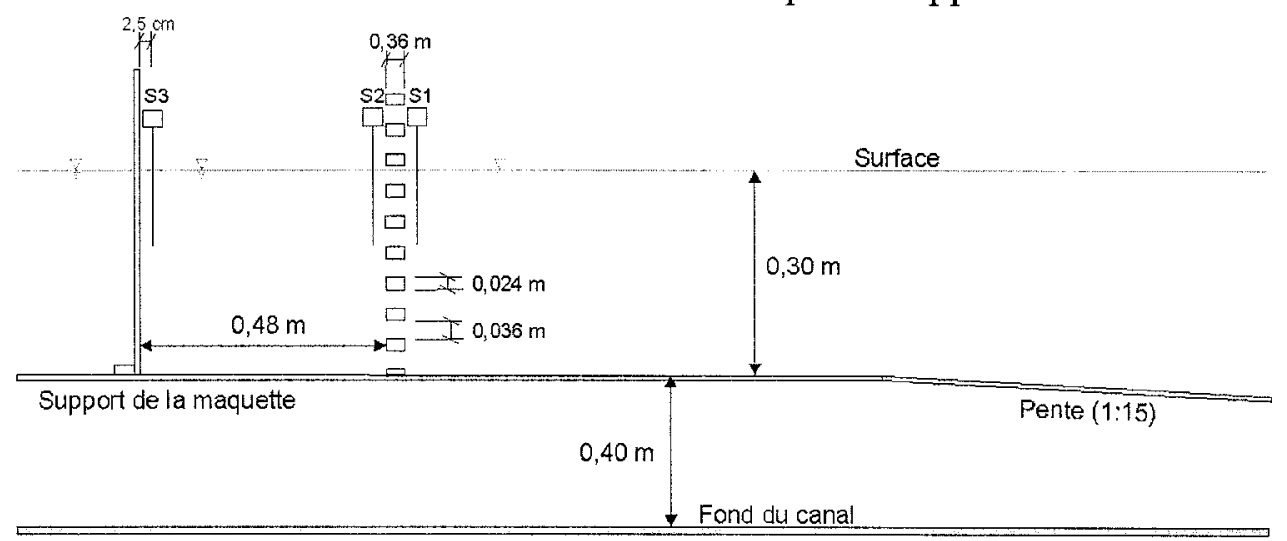

Figure 3. Principales dimensions de la maquette et du canal. Main dimensions of the model and the wave flume.

La source laser disponible lors de nos expériences est un Spectra-Physics à Argon ionisé produisant un faisceau continu d'une puissance maximale de $15 \mathrm{~W}$. Le faisceau lumineux du laser est guidé par une fibre optique jusqu'au dispositif d'illumination situé sous le canal. 
Ce dispositif est constitué d'un miroir et d'une lentille de collimation déviant le faisceau lumineux incident sur une des facettes d'un miroir octogonal rotatif. Le faisceau réfléchi par la facette est dirigé sur un miroir concave le déviant à son tour verticalement (Figure 4). La rotation d'une facette (d'un 1/8 de tour) permet au rayon lumineux de balayer la surface du miroir concave et de générer une (pseudo) nappe laser verticale large de $0,50 \mathrm{~m}$ pour une épaisseur de $5 \mathrm{~mm}$ (Gray et al., 1991, Jensen et al., 2001). La vitesse de rotation du miroir contrôle la période de balayage du faisceau laser d'un côté à l'autre de la nappe. Cette période varie entre 0,5 et $20 \mathrm{~ms}$ selon le type d'exposition désiré (illumination unique ou multiple des particules sur l'image).

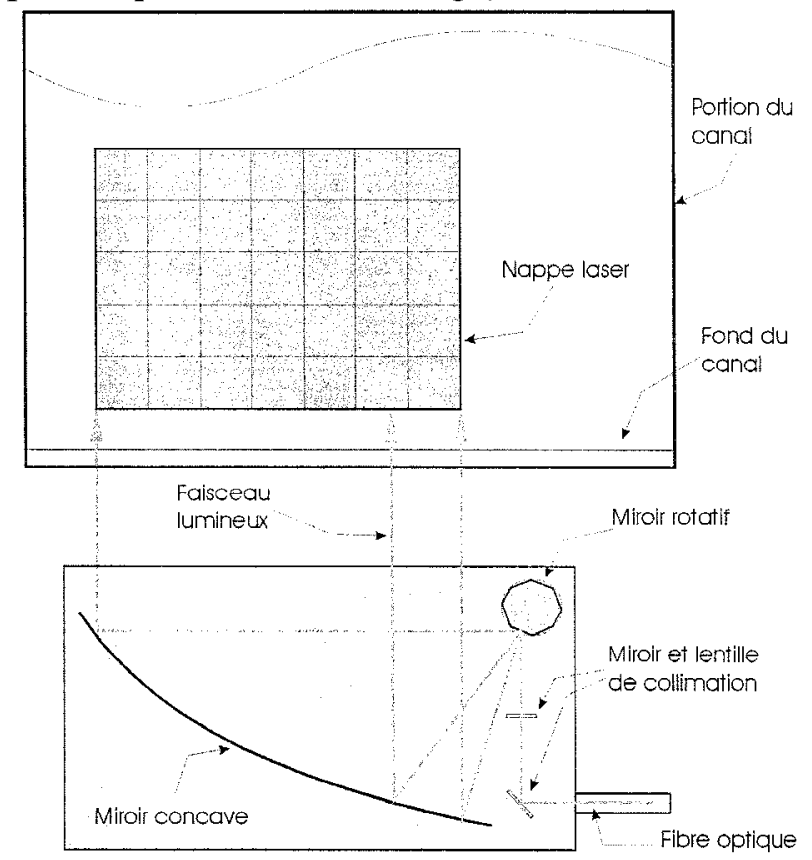

Figure 4. Dispositif d'illumination laser par miroir rotatif. Laser illumination using scanning beam illumination system.

Ce procédé génère une nappe laser plus large que celle habituellement produite par un laser pulsé mais la faiblesse de son intensité ainsi que sa répartition spatiale (gaussienne au lieu de rectangulaire pour le $\mathrm{Nd}$ :Yag) conduisent cependant à restreindre son emploi à l'étude d'écoulements à faible vitesse.

L'acquisition vidéo est réalisée par une camera numérique 12 bits Kodak MegaPlus ES1, dotée d'un capteur CCD de $1008 \times 1016$ pixels et d'un objectif photo $\mathrm{f}_{\#}=50 \mathrm{~mm}$ (ouverture 2). La commande et le transfert des données de cette caméra sont effectués par le processeur PIV 2100 de Dantec Dynamics. Nous utilisons la caméra dans son mode "single frame" à la fréquence de $25 \mathrm{~Hz}$, soit une image toutes les $40 \mathrm{~ms}$. Chacune est exposée pendant $4 \mathrm{~ms}$.

\subsection{Adaptation du dispositif de PIV aux écoulements de houle}

L'écoulement étudié étant périodique, il est nécessaire de synchroniser les prises de vues à une référence temporelle liée aux oscillations de la surface libre. Dans ce but, une carte d'acquisition enregistre, en continu et à $100 \mathrm{~Hz}$, les signaux 
provenant des sondes limnimétriques placées dans le canal. Au passage par zéro croissant du signal de la sonde S1 (zero-up-crossing), le dispositif d'acquisition de la surface libre transmet au système de PIV un signal TTL (passage de 0 à 5 V) qui déclenche une séquence de prises de vue. Cette séquence est constituée de $n$ images, $\mathrm{n}$ étant l'entier inférieur au produit de la période de houle par la fréquence des images (pour $\mathrm{T}=1,3 \mathrm{~s}$ et 25 images/s, $\mathrm{n}=32$ ). Ce dispositif de synchronisation permet ainsi d'enregistrer une série de plusieurs périodes de houle.

Le nombre de séquences est limité essentiellement par les contraintes de conservation des images $(0,97$ Mo chacune) et par la baisse de la densité de l'ensemencement.

\subsection{Méthode d'analyse de l'écoulement par PIV}

Le traitement des images est réalisé dans l'environnement Matlab par un ensemble de routines développées initialement par J.K. Sveen ${ }^{1}$. Nous les avons modifiées afin de les adapter à nos contraintes expérimentales et pouvoir intégrer de nouveaux algorithmes.

Ces travaux sont illustrés par les mesures de vitesse dans la chambre du caisson perforé pour la période $1,3 \mathrm{~s}$. Les figures $5 \mathrm{a}$ et $\mathrm{b}$ montrent les champs des vitesses obtenus après l'analyse par PIV. La paroi perforée se trouve à la droite du champ et la paroi arrière à sa gauche. A l'arrière plan est insérée l'image correspondant au calcul du champ (image 1).

L'analyse des images est basé sur un processus itératif avec une diminution progressive de la taille des fenêtres d'interrogation (Westerweel et al, 1997). Afin de déterminer les tendances générales de l'écoulement lors de la première passe, nous employons une fenêtre d'interrogation relativement grande $(128 \times 128 \mathrm{px})$, de taille et de position identique sur l'image 1 et sur l'image 2. Afin de couvrir la surface totale de l'image 1 et prendre en compte le maximum de particules, les fenêtres sont décalées les unes par rapport aux autres avec un recouvrement de $50 \%$.

La deuxième passe met en œuvre une fenêtre d'interrogation moitié moins grande (soit $64 \times 64 \mathrm{px}$ ). Chaque fenêtre de l'image 2 est translatée par rapport à sa correspondante de l'image 1 en fonction du précédent champ estimé des déplacements. Ce champ est auparavant corrigé des éventuels déplacements erronés puis interpolé afin de s'adapter à la réduction de taille des fenêtres. La troisième passe est sur le même principe que la seconde et nous terminons donc l'analyse avec une fenêtre d'interrogation de $32 \times 32 \mathrm{px}$.

La figure de gauche (5a) donne la cinématique de l'onde lors du passage par le niveau moyen montant (à la paroi perforée). Le remplissage de la chambre est réalisé par l'eau entrant avec des vitesses horizontales fortes au niveau des perforations $\left(\left|\mathrm{u}_{\max }\right|=0,246 \mathrm{~m} / \mathrm{s}\right)$. L'accroissement de volume du fluide provoque la montée de l'eau le long du mur arrière $\left(\mathrm{v}_{\max }=0,207 \mathrm{~m} / \mathrm{s}\right)$.

La figure de droite $(5 \mathrm{~b})$ correspond à un instant après le passage de la crête à travers la paroi perforée. L'abaissement du niveau de la surface libre devant la

${ }^{\mathrm{I}}$ Toolbox MatPIV, Université d'Oslo, site internet www.math.uio.no/ jks/matpiv 
paroi perforée entraîne un phénomène de vidange de la chambre comme l'indiquent les vecteurs. Sur cette figure, les vitesses maximales horizontales et verticales sont : $u_{\max }=0,147 \mathrm{~m} / \mathrm{s}$ et $\left|v_{\max }\right|=0,123 \mathrm{~m} / \mathrm{s}$.
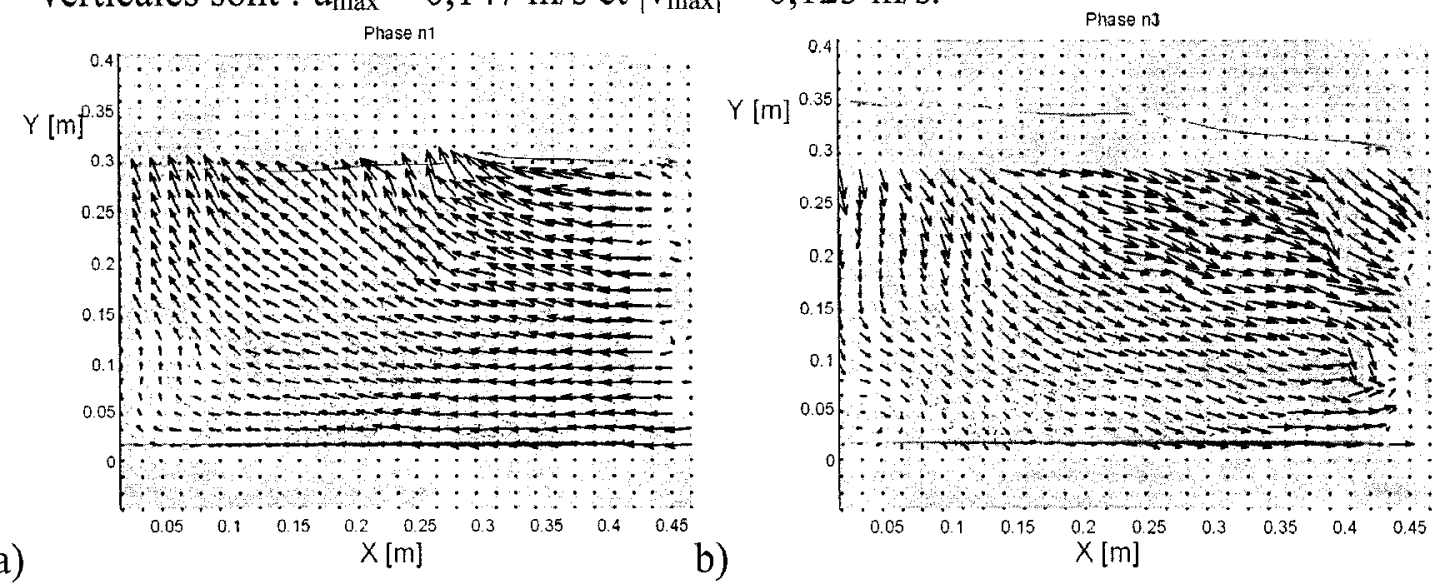

Figure 5. Exemples de champs des vitesses dans la chambre du caisson. Examples of caisson chamber velocity fields.

A l'issue de la dernière passe, les déplacements sont évalués à $\pm 1 / 2 \mathrm{px}$. En effet, dans la configuration de l'expérience présentée ici, le diamètre des particules de pollen de pin utilisées pour l'ensemencement correspond à un peu moins de deux pixels. La méthode conduisant à une résolution inférieure au pixel atteint donc sa limite d'utilisation. Cette incertitude sur le déplacement conduit à estimer les vitesses réelles du fluide à $\pm 6 \mathrm{~mm} / \mathrm{s}$, soit environ 3 à $5 \%$ des vitesses maximales précédemment évoquées.

Cette valeur peut paraître importante par rapport à celle obtenue avec la VDL mais cette dernière méthode repose sur une mesure absolue de la vitesse en un seul point (effet Doppler). La PIV conduit à une mesure relative des vitesses, elle est donc plus sujette aux imprécisions engendrées par les distorsions optiques, le comportement des particules, la méthode de traitement. Nous sommes néanmoins dans un cas extrême, l'étude d'une zone de l'écoulement plus restreinte pouvant réduire l'incertitude d'un facteur 10.

L'avantage principal de la PIV sur la VDL réside dans l'acquisition du champ des vitesses instantanées sur une portion très étendue de l'écoulement : la zone de mesure est de $0,48 \times 0,30 \mathrm{~m}$ pour les figures 7 et comporte près de 600 points. De plus la cinématique de l'écoulement est obtenue de façon globale sans reconstitution préalable d'une vitesse moyenne en chaque point (Tabet Aoul et Bélorgey, 1998). D'autre part les résultats sont obtenus rapidement : quelques dizaines de secondes pour l'acquisition des images et quelques dizaines de minutes pour le traitement des séquences.

\section{Conclusion}

Nous avons présenté quelques unes des méthodes d'analyse des images mises en œuvre dans le cadre de la vélocimétrie par imagerie de particules. Cette technique de mesure est adaptée aux études du génie côtier car elle permet de connaître le champ des vitesses sur une portion étendue des écoulements. Elle peut également être pratiquée à l'aide de moyens expérimentaux déjà présents dans les laboratoires (laser 
Argon ionisé, caméscope,...) pour un coût relativement modeste. Ces deux arguments devraient par ailleurs encourager l'usage de la PIV en génie côtier.

\section{Remerciements}

Ces travaux de recherche sont réalisés dans le cadre d'une Bourse Individuelle Marie Curie du programme "Potentiel Humain" de la Commission Européenne (contrat HPMF-2000-01010 du $5^{\text {ème }}$ PCRDT). La Commission Européenne n'est pas responsable des résultats ou des opinions exprimés dans cet article.

\section{Bibliographie}

Adrian R J (1984). "Scattering particle characteristics and their effect on pulsed laser measurements of fluid flow: speckle velocimetry vs. particle image velocimetry", Applied Optics, vol 23, pp1690-1691

Adrian R J (1991). "Particle-imaging techniques for experimental fluid mechanics", Annual Review: Fluids Mechanics, vol 23, pp 261-304

Huang H T, Fiedler H E et Wang J J (1993). "Limitation and improvement of PIV. Part II: Particle image distorsion, a novel technique", Experiments in Fluids, vol 15, pp 263-273

Jensen A, Sveen J K, Grue J, Richon J-B et Gary C (2001). "Accelerations in water waves by extended particle image velocimetry", Experiments in Fluids, vol 30, pp 500-510

Lauterborn W et Vogel A (1984). "Modern optical techniques in fluid mechanics", Annual Review: Fluids Mechanics, vol 16, pp 223-244

Raffel M, Willert C et Kompenhans J (2001). "Particle Image Velocimetry: a practical guide (3rd edition)", Springer (Berlin), $253 \mathrm{p}$

Tabet Aoul E H et Bélorgey M (1998). "Etude de la circulation à l'intérieur du caisson Jarlan", $V^{\text {èmes }}$ Journées Nationales de Génie Civil - Génie Côtier, Toulon, pp 329-336.

Westerweel J (1997). "Fundamentals of digital particle image velocimetry", Measurement Science and Technology, vol 8, pp 1379-1392

Westerweel J, Dabiri D et Gharib M (1997). "The effect of a discrete window offset on the accuracy of cross-correlation analysis of digital PIV recordings", Experiments in Fluids, vol 23, pp 20-28

Willert C E et Gharib M (1991). "Digital particle image velocimetry", Experiments in Fluids, vol 10, pp 181-193 